was due to the increase in the time of presence ( $\left.\mathfrak{t}^{\prime}\right)$, and not due to the increase in the number of immigrants $(\nu i m)$.

Another example: in most cases both in the field and in the controlled laboratory experiments the number of young is lower than the number of adults. This results from the fact that a young individual (arbitrarily, up to seven weeks old) can be present at most seven weeks, and then it becomes adult; as an adult it can be present for 18 months.

\title{
5.2. Numbers - Methods of Assessment
}

\section{Henryka CHEŁKOWSKA \& Jacek GOSZCZYŃSKI}

\subsubsection{Capture Techniques}

The methods for the assessment of bank vole numbers do not differ from those applied for number estimation of other small rodents. Thus we will only enumerate them here, and indicate what additional information we can get using these methods, without going deep into their advantages and shortcomings. Those interested in the technique of calculations or in the comparison of various methods are referred to Smith et al. (1975).

The cases when numbers or density can be determined by the removal of all or almost all individuals living in a given area are rare and limited to isolated populations, e.g. on islands (Andrzejewski et al., 1967; Gliwicz et al., 1968), artificially isolated (Gębczyńska, 1966), or confined. Long-term trapping from open areas can lead to the so-called complete removal, despite disturbance caused by immigrants, but the extent of the "vacuum" produced is not exactly known (Andrzejewski \& Wrocławek, 1962; Kozakiewicz, 1976).

Indirect methods such as tracking on snow provide only rough approximations of vole numbers as the activity of these animals on the surface of snow cover largely depends on weather and snow quality (Rotshild, 1959). Strongly ramified burrow systems of the bank vole do not allow the application of flooding (Truszkowski, personal communication). For these reasons we estimate numbers from samples of individuals caught in a given location. Most frequently, bank voles are caught on sampling areas or on trap-lines with a specific configuration 
of live or snap traps, depending on the purpose of the study. Using live traps and the CMR method, that is, marking the individuals caught and releasing them, we can get much more information on the population than using snap traps. Detailed analyses of natality and mortality, spatial and social organization, mechanisms of number regulation, etc. are usually possible only when live trapping is used on study plots. This is not the case when we want to collect material for mortality and natality estimates, or to analyse the diet. However, in many cases snap traps are used for economical reasons (lower costs of the study in terms of labour and money).

Good results in catching bank voles can be obtained by using cones or cylinders (Andrzejewski \& Wrocławek, 1963); Chełkowska, 1967), though in some cases live traps can be more efficient (Andrzejewski \& Rajska, 1972).

Spacing of traps (distances between traps) largely affect the estimates of bank vole densities, particularly when live trapping is used (Tana$\mathrm{ka}, 1966)$. There are many papers discussing the optimal distance between traps (e.g. Smith et al., 1969/1970; Wheeler \& Calhoun, 1967: quoted by Smith et al., 1975). This problem has not been ultimately solved, however, and according to Smith et al., (1975), the recently used distance of $15 \mathrm{~m}$ is satisfying. Pelikàn (1974) recommends a closer spacing to intensify trapping in the areas where prebaiting is not used.

The intensity of trapping (the number of trap checking per day, and the number of trapping days per week) is of great importance, especially when the calender of catches is used to estimate density. A very intense live trapping can produce a group of animals excluded from their normal life in the population, and "living" in traps, that is, recaptured immediately after release (Andrzejewski et al., 1967). In addition, these animals block the access to the trap for other individuals. When trapping is carried out at excessively long time intervals, the animals present for a short time in the area cannot be trapped. This may be the case of young individuals, which show higher migration and mortality rates than adults. According to some authors, the presence of trap-shy individuals in the population can account for some errors in density estimates (Kikkawa, 1964; Bailey, 1968; Andrzejewski et al., 1971). These errors can be reduced by using the calendar of catches or analysing not only current catches at a given time but also the earlier and the subsequent catches (Fig. 5.3).

The rate of capture is greatly affected by the number of traps per point or station (Andzzejewski et al., 1966). Most frequently one or two traps are used per pcint, though on transects more traps per point would be needed to ensure a high rate of catches. 


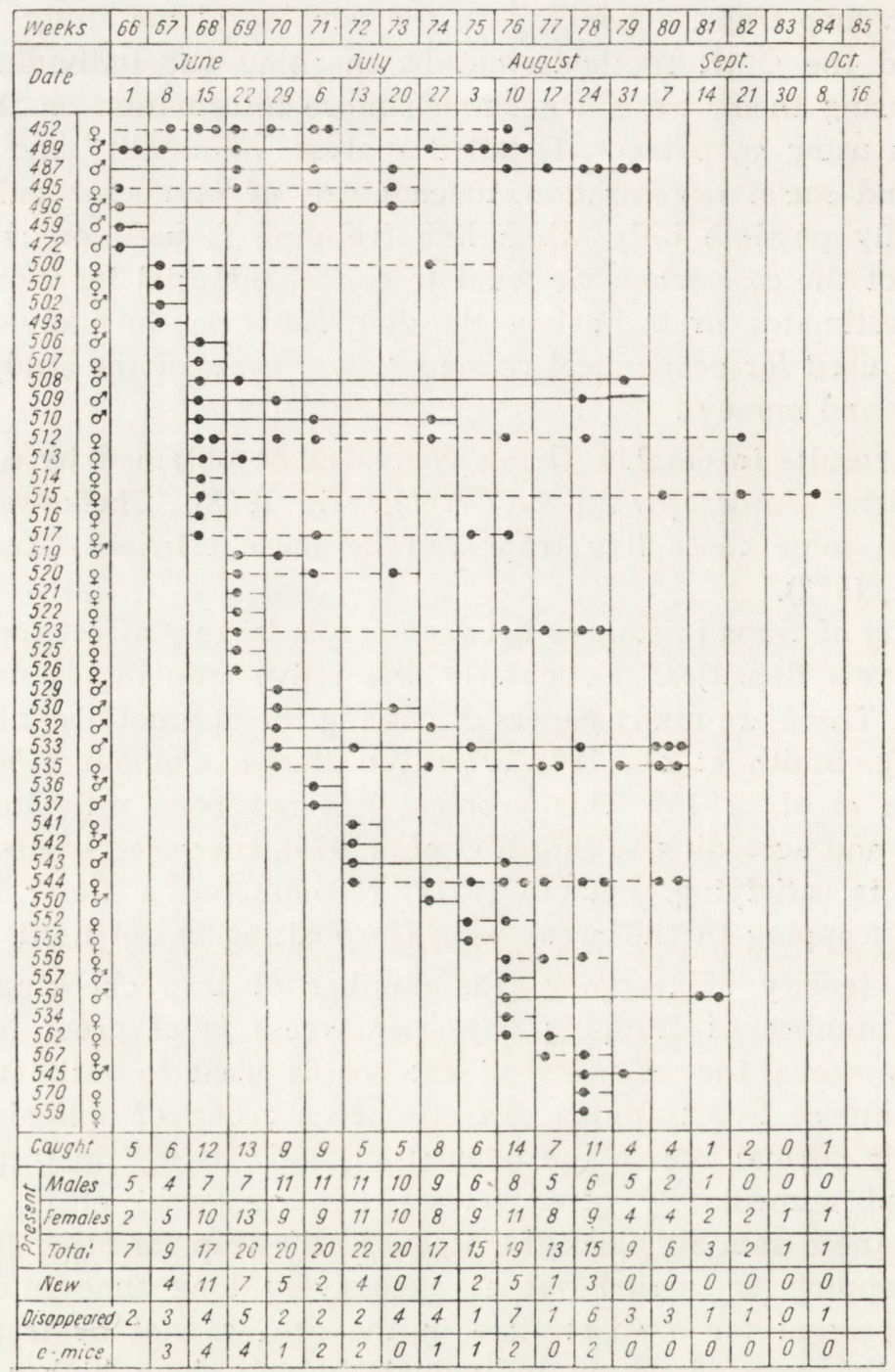

Fig 5.3. Calendar of catches (modified from Petrusewicz and Andrzejewski, 1962). Dots represent catches, the line connecting the dots - duration of trap presence in the study areas. Horizontal data: Life history of an individual. Points indicating captures can be replaced with numbers indicating the location of trapping points (e.g. A1, A2, B7, etc.) which allows determination of the area covered by individual animals. Weight and increase in weight are obtained for each time period. Length of stay $\left(t^{\prime}\right)$, average distance between catches (= reciprocal of real trappability), etc. can also be calculated. Vertical data: the situation at a given moment e.g. how many individuals were caught, how many are known to be present (number of lines depicts the number of individuals present on a given day), how many were newly caught, how many males or females, how many migrants, how many disappeared, etc. 
Baiting obviously increases the rate of capture (Andrzejewski \&. Wrocławek, 1963; Tanton, 1969; and others), and facilitates the subsequent estimation of the number of animals living in the area (Gentry et al., 1971), but at the same time it accounts for the concentration of animals at the edge of the study plot, and disturbs the spatial organization of the population by increasing the carrying capacity of this area (Pelikàn, 1974). The additional food can change population dynamics in the case of live-trapping (Gentry, 1968; Smith, 1971), and it increases the distance from which animals are trapped when snap traps are used.

\subsubsection{Study Plots}

So far study plots of various sizes have been used. The most widely applied method has been Standard Minimum (Grodziński et al., 1966). It was used in the IBP studies on the productivity of small mammal populations (Pucek \& Ryszkowski, 1970). The uniform method of capture (the plot of a side length of $225 \mathrm{~m}$, with a network of traps $15 \mathrm{~m}$ apart, a fixed time of baiting and trapping) allows a comparison between different populations and ecosystems. A plot of this size ensures rapid capture of all animals, and a sufficiently large material to estimate ecological parameters (e.g. reproductive rate, age structure of the population, etc.). At the same time, it covers at least several individual home ranges, which allows an alternate application of the removal and CMR techniques.

According to many authors, however, the size of the Standard Minimum limits its application. The reasons are that setting and checking traps on such an area is labour consuming, and frequently it is difficult to find a homogeneous habitat of this size. Many authors argue that similar results can be obtained using smaller areas (Myllymäki, et al., 1971; Pelikán, 1971; Zejda \& Holišova, 1971). On smaller plots (less than $1 \mathrm{ha}$ ), however, estimates of the number of resident individuals are more biased (Chełkowska \& Ryszkowski, 1966). It is also difficult to calculate the number of individuals in the study area, particularly if there is no baiting. This is related to a lower rate of capture and some disturbances during the capture (Pelikán, 1974; Hansson, 1975). In addition, density estimates based on individual home ranges can be misleading due to a high mobility of rodents (Adamczyk \& Ryszkowski, 1968; Tanton, 1969; Ryszkowski, 1971) if the plot is too small.

But if we do not want to catch a large number of animals, and instead are interested in quick results at low costs, small plots seem best. For these reasons they are frequently used. In Scandinavia, the Small Quadrat method is used to predict rodent numbers (Myllymäki 
et al., 1971; Hansson, 1974a). In this method the study plot is reduced to a quadrat $15 \mathrm{~m}$ per side, with three snap traps in each corner. A modified Standard Minimum method (with a reduced surface area) was used by Hansson (1975) and Ryszkowski et al. (1973). Also Pelikán (1974) used small plots.

As plots of different sizes are used, a comparison of the results obtained seems badly needed. Hansson (1975) has done this for the Small Quadrat and Standard Minimum. He developed regression equations for particular species that allow density estimates from small quadrats.

When captured animals are removed from a plot, the simplest way of estimating numbers is to divide the number of individuals caught by the plot size. As the duration of the trapping period significantly influences the results, usually a linear regression is used to estimate the number of animals on the plot (De Lury, 1947; Hayne, 1949), or the maximum likelihood method proposed by Janion et al. (1968) that allows estimation of an average probability of capture. This method of calculation makes it possiblel to estimate the total number of individuals without removing them for many days.

When live trapping, the number of animals can be estimated by means of the calendar of catches (Blair, 1951; Davis, 1955; Adams, 1959; Petrusewicz \& Andrzejewski, 1962). Due to the interpolation of date to the periods between catches (Fig. 5.3), this method enables us to determine the composition of the population at any time instant or time period, and to follow the fates of individual animals.

Also, a curvilinear regression can be used (Tanaka \& Kanamori, 1967). The densities estimated in this way are of relative character, and they can be used for the analysis of population dynamics of the bank vole, comparison of numbers in different ecosystems, etc., thus when we want to record time and habitat-related changes.

\subsubsection{Trapping Range}

It is known, however, that the area from which the animals are trapped is larger than the area covered with traps. Animals caught on the plot consist of individuals spending all their time within the plot (obviously, if the plot is large enough to cover an average home range), and of the animals only partly active within the plot (Calhoun \& Casby, 1958), as well as migrants. If baiting is used, residents from beyond the plot and probably more migrants are captured (Pelikán et al., 1964; Chełkowska \& Ryszkowski, 1967). Influx of animals into the plot leads to the so-called edge effect, which is revealed by increased catches of traps located at the plot edge. 
Thus, to get an absolute estimate of density, it is necessary to calculate range of trapping. It seems that the methods of rejecting the outer line of traps, and using only the inner square of traps for density estimation (Adamczyk \& Ryszkowski, 1968; Pelikán, 1969) are now considered as relative methods. More promising are the methods based on estimating the size of the area from which animals are removed by the traps.

Some authors suggest that a belt of the width equal to the average diameter of the home range or at least to the half of it should be added to the plot area (Dice, 1938; Pelikán, 1974). Such estimates raise some reservations. For example, they should be preceded by gathering information on the average distance covered by individuals, and this parameter is not easier to determine than density itself. The methods for estimating the range of the influence exerted by the trapping area given by Hansson (1969b) and Smith et al. $(1969,1970)$ are based on delimitation of the so-called inner square within which the distribution of catches per traps is homogeneous. Smith et al. $(1969,1970)$ compare the frequency of catches for each belt of traps. Usually the highest frequency of catches was recorded in the first belt of traps; it decreased towards the centre of the plot until a stable level was reached. According to Smith et al. (1969/1970), the breadth of the boundary area, that is, the zone with increased catches, should be added to the area of the study plot. Hansson (1969b) calculated the range of the influence exerted by the plot assuming that the ratio of the number of animals caught on the whole sampling area to the size of this area increased by the boundary zone equals the ratio of the number of animals caught in the inner square to the size of this square. The inner square in this method was determined according to Pelikán (1969).

To estimate the trapping range American workers propose the use of assessment lines on which animals are caught when the catches on the basal plot are completed. Cumulating the catches from the end of such a line and analysing the shape of the curve, it is possible to find the boundaries of the complete removal and the range of the incomplete removal (Smith et al., 1971). Assessment lines are also recommended to determine the range of the plot when the CMR method is used (Nabkolz, 1973 quoted by Smith et al., 1975; Swift and Stenhorst, 1976). These last authors use assessment lines to determine the ratio of marked to unmarked individuals at different distances from the plot.

The range of the influence of the plot can also be estimated by using coloured baits prepared according Holišova's (1968) method, which after capture of animals, are detected in alimentary canals of rodents (Ryszkowski, 1971). Using this method, Ryszkowski estimated the 
number of animals in the central and peripheral parts of the plot. To determine the range of removal, he assumed that both the whole plot and the central part (inner quadrat) are visited by individuals from identical distances. An average distance of removal estimated in this way is higher than that obtained by the Hansson method (Ryszkowski, 1971). This method can also be used in vivo as coloured baits can be identified in faeces of rodents (Goszczyński, 1971).

\subsubsection{Methods for Estimating Densities}

The concepts of absolute and relative numbers are widely known in ecology. Absolute numbers can be characterized as the number of individuals per unit area, while the relative numbers denote the number of individuals recorded by means of comparable methods with no possibility to relate these numbers to the size of the area from which the animals were captured. Absolute numbers are needed in the studies of productivity, trophic relations, and determination of population indices. Relative numbers are used for all other estimates that need not be particularly accurate. First of all they are of comparable value.

Tra p-lines. To estimate relative numbers, the method of "traplines" can be used. In this method traps are evenly spaced along lines, the number of traps per point being constant. It is difficult to capture all the animals when this method is used (Calhoun, 1949; Hansson, 1967b). Without calculating the total number of animals occurring in the area crossed by the line, the comparison of results from different lines can be misleading. Though this method is easy to apply, it is not possible to get absolute density estimates from the lines alone, when no additional estimates of the range of animal activity are available (Brandt, 1962). Even lines with variable distances between traps do not allow the calculation of home ranges because the number of rodents caught is disproportionately high in places of trap concentration (Hansson, 1974b). Hence results obtained from trap-lines are often expressed in terms of the number of individuals per trap per day. Obviously, these are relative estimates and to get comparable results for different habitats or years, the trap-lines should be standardized, that is, the number of capture points, the number and the type of traps, time of exposure, bait, etc. should be identical for various capture series. Although there are statistics allowing analysis of material collected from non-standardized lines (Linn \& Downton, 1975), they can lead to errors. Moreover, results obtained by Ryszkowski (1971), who found that bank voles are more mobile in spring, when densities are low, than in autumn, show that the trap line method is likely to obliterate seasonal differences in population dynamics. Obviously, the results also depend on weather 
during trapping (Sidorowicz, 1960). Nikitina (1958, 1961b), who compared results from trap lines with those from plots for marked animals, found that number indices from lines are markedly less variable than the actual population density. Russian researchers, however, have for many years used mostly capture lines as the best method to get a quick characteristic of different habitats with respect to their rodent populations. Due to the standardized baiting, traps, and trap spacing, the results are comparable, and are presented in terms of the number of animals caugth per 100 trap-days (Kučeruk, 1963; Popov, 1966).

Attempts to estimate absolute numbers $(N)$ from transects are presented by Smith et al., (1971) and Kaufman et al., (1971). They captured rodents from the census line first, and then from the assesment line. When one census line is used, the assesment line should cross it at an acute angle, and the capture period should be short (Smith et al., 1971). Although this cross line method is simple, and small areas can be sampled, its application is limited because of the variability of enviromental conditions and a rapid immigration of animals (see Smith et al., 1975). An attempt was made to overcome these difficulties by simultaneous application of several cross lines (Kaufman et al., 1971), and calculating a joint density. In practice, the lines were grouped in an octagon, according to the recommendations of Wheeler \& Calhoun (1968), and for estimating the range, a line of traps crossing this octagon was used. The results obtained, however, are not unequivocal (Gentry et al., 1971b; Kaufman et al., 1971), since immigration of animals into the octagon made it impossible to estimate the range (Gentry et al., 1971b).

This review of the methods applied, though incomplete, may provide a basis for selection of the best one in view of the study purpose and time/financial limits. We do not suggest any choice but we postulate that the authors should describe in detail the methods they use so that future results on vole densities in various habitats and years may be compared with greater reliability. 